Research Article

\title{
Topological Indices of Pent-Heptagonal Nanosheets via M-Polynomials
}

\author{
Hafiza Bushra Mumtaz, ${ }^{1}$ Muhammad Javaid ${ }^{1},{ }^{1}$ Hafiz Muhammad Awais, 1 \\ and Ebenezer Bonyah iD ${ }^{2}$ \\ ${ }^{1}$ Department of Mathematics, School of Science, University of Management and Technology (UMT), Lahore, Pakistan \\ ${ }^{2}$ Department of Mathematics Education, Akenten Appiah-Menka University of Skills Training and Entrepreneurial Development, \\ Kumasi 00233, Ghana
}

Correspondence should be addressed to Ebenezer Bonyah; ebbonya@gmail.com

Received 16 September 2021; Accepted 20 October 2021; Published 12 November 2021

Academic Editor: Ali Ahmad

Copyright $(92021$ Hafiza Bushra Mumtaz et al. This is an open access article distributed under the Creative Commons Attribution License, which permits unrestricted use, distribution, and reproduction in any medium, provided the original work is properly cited.

\begin{abstract}
The combination of mathematical sciences, physical chemistry, and information sciences leads to a modern field known as cheminformatics. It shows a mathematical relationship between a property and structural attributes of different types of chemicals called quantitative-structures' activity and qualitative-structures' property relationships that are utilized to forecast the chemical sciences and biological properties, in the field of engineering and technology. Graph theory has originated a significant usage in the field of physical chemistry and mathematics that is famous as chemical graph theory. The computing of topological indices (TIs) is a new topic of chemical graphs that associates many physiochemical characteristics of the fundamental organic compounds. In this paper, we used the M-polynomial-based TIs such as 1st Zagreb, 2nd Zagreb, modified 2nd Zagreb, symmetric division deg, general Randi $c$, inverse sum, harmonic, and augmented indices to study the chemical structures of pent-heptagonal nanosheets of $V C_{5} C_{7}$ and $H C_{5} C_{7}$. An estimation among the computed TIs with the help of numerical results is also presented.
\end{abstract}

\section{Introduction}

Nanostructures [1, 2] have been studied as new materials with the size of elementals structures that has been engineered at the nanometers' scale. Most of the materials in this size range usually show novel behavior. Therefore, intervention in the characteristics of structures at the nanoscale allows the formation of devices and nanomaterials with completely or enhanced novel functionalities and properties. Understanding the science of nanostructures is curiosity and important driven not only for the interesting nature of the topic but also for novel and overwhelming usage of nanoscale systems in various fields of science and technology. Nanotechnology can be recognized as a technology of design, application, and fabrication of nanomaterials, and nanostructures [3].

The branch of nanotechnology and nanoscience is being perused by chemists, physicists, materials scientists, engineers, biologists, computer scientists, and mathematicians [4]. So, it is also interdisciplinary. Nanostructures may be divided based on modulation and dimensionality. Most of the distinct nanotubes, zeolites, aerogel, core-shell structure, and nanoporous materials have unique properties. $\mathrm{Nu}-$ merous techniques have been utilized for the synthesis of nanomaterials with no. of degrees of success, and several direct as well as indirect methods are used for their properties [5]. The motivation to develop the nanomaterials is that the characteristics become size based in the nanometer range due to quantum confinement effect and surface effect. The chemical bonds, magnetic properties, geometric structure, electronic properties, ionization potential, mechanical strength, optical properties, and thermal properties are affected due to particle size in nanometers range. Nanostructures show characteristics mostly higher than the conventional coarse-grained material. These contain hardness/increased strength, toughness/improved ductility, 
enhanced diffusivity, reduced density, higher electrical resistance, reduced elastic modulus, lower thermal conductivity, increase specific heat, higher thermal expansion coefficient, increased oscillator and strength luminescence, blue shift absorption, and superior soft magnetic characteristics in comparison to the conventional bulk material. Furthermore, these characteristics are being briefly examined to discover new tools. The interesting branch of nanotechnology has a vast range of different types of applications. The use of nanomaterials has manufactured transistors having low speed and laser having low threshold current. These are utilized in satellite receivers having low noise amplification as a source for fiber optics communications and compact disk player systems. Constructive tools of nanostructures contain UV-resistant wood coating and self-cleaning glass. On the other hand, nanoscale tools are being utilized in the field of medicine for the prevention and treatment of diseases, diagnosis, and in magnetic resonance imaging, drug delivery system, radioactive tracers, etc. [6]. The importance of nanomaterials is rising nowadays. Many other types of tools may be possible with the peculiar and novel characteristics of nanomaterials $[7,8]$.

Therefore, TIs are useful to define molecular nanomaterials. Nanostructures, that have a scale of less than $100 \mathrm{~nm}$, contain nanosheets, nanotubes, and nanoparticles. Nanosheets (two-dimensional nanomaterials) have a sharp edge and large surface area that cause them to play a vital role in various types of tools such as catalysis, energy storage bioelectronics, and optoelectronics [9, 10]. Silicone, borophene, and graphene are specific nanosheets. Due to the rare optical, electrical, mechanical, and structural characteristics, graphene nanosheets received great recognition from industrial and academic researchers [11]. The different properties of the $C_{5} C_{7}$ nanosheet have become the most advanced field in research. A $C_{5} C_{7}$ structure is developed by alternating $C_{5}$ and $C_{7}$ [7]. In 2009, Graovac et al. studied the GA index of $T U C_{4} C_{8}$ (S) nanotubes. In 2011, Graovac et al. [12] studied the fifth geometric arithmetic index for nanostar dendrimers, and Asadpour et al. calculated, Zagreb, Randi $c$, and $\mathrm{ABC}$ indices of $T U C_{4} C_{8}(\mathrm{R})$ and $T U C_{4} C_{8}(\mathrm{~S}) \mathrm{V}$-Phenylenic nanotorus and nanotubes. In 2014, Al-Fozan et al. solved Szeged index of H-naphthalene nanosheets $(2 \mathrm{n}, 2 \mathrm{~m})$ and $C_{4} C_{8}(\mathrm{~S})$. Loghman and Ashrafi studied the Padmakar-Ivan (PI) index of $T U C_{4} C_{8}(\mathrm{~S})$ nanotubes. For further discussion, see [13-15].

However, the combination of three fields such as mathematics, physical chemistry, and information sciences lead to a modern field known as cheminformatics [16-18]. It develops a mathematical relationship between a property and structural attributes of different types of chemicals called by quantitative-structures' activity and qualitative-structures' property relationship that are utilized to forecast the organic sciences and biological properties in the field of engineering and technology [19, 20]. Graph theory has originated a significant usage in the field of mathematical chemistry that is famous as chemical graph theory.

Polya gave the idea for counting polynomials in the field of chemistry [21], and Wiener introduced the concept of TI related to the paraffin's boiling point [22]. Computing the
TIs is a new field of chemical graphs that associates many physiochemical characteristics of the fundamental chemical compounds [23-27].

\section{Preliminaries}

A molecular structure $\Gamma=(V(\Gamma), E(\Gamma)) ; \quad V(\Gamma)=\left\{s_{1}, s_{2}\right.$, $\left.s_{3}, \ldots, s_{n}\right\}$ and $E(\Gamma)$ are nodes (vertices) and edge set of $\Gamma$. $|V(\Gamma)|=v$ and $|E(\Gamma)|=e$ is the order and size of $\Gamma$. In a connected and simple molecular graph, a path is represented within two vertices and the distance between the two vertices $s$ and $t$ is mentioned as $\varphi(s, t)$, in a graph $\Gamma$, see [28-30]. In this paper, a graph is connected and simple, having no multiple edges or loops.

1st and 2nd Zagreb indices: let $\Gamma$ be a molecular structure; then, its 1st and 2nd Zagreb indices [31] are

$$
\begin{aligned}
& M_{1}(\Gamma)=\sum_{s \in V(\Gamma)}[\varphi(s)]^{2}=\sum_{s t \in E(\Gamma)}[\varphi(s)+\varphi(t)], \\
& M_{2}(\Gamma)=\sum_{s t \in E(\Gamma)}[\varphi(s) \times \varphi(t)] .
\end{aligned}
$$

General Randi $c$ index: if $R$ is the real number, $\alpha \in R$, and $\Gamma$ is a molecular structure, the general Randi $c$ index [32] is

$$
R_{\alpha}(\Gamma)=\sum_{s t \in E(\Gamma)}[\varphi(s) \varphi(t)]^{\alpha} .
$$

Symmetric division deg index: for a molecular structure $\Gamma$, the symmetric division deg index [33] is

$$
\operatorname{SDD}(\Gamma)=\sum_{s t \in E(\Gamma)}\left[\frac{\min (\varphi(s), \varphi(t))}{\max (\varphi(s), \varphi(t))}+\frac{\max (\varphi(s), \varphi(t))}{\min (\varphi(s), \varphi(t))}\right] .
$$

Harmonic index: for a molecular structure $\Gamma$, the harmonic index [34] is

$$
H(\Gamma)=\sum_{s t \in E(\Gamma)} \frac{2}{\varphi(s)+\varphi(t)} .
$$

Inverse sum index: for a molecular structure $\Gamma$, the inverse sum index [35] is

$$
\operatorname{IS}(\Gamma)=\sum_{s t \in E(\Gamma)} \frac{\varphi(s) \varphi(t)}{\varphi(s)+\varphi(t)} .
$$

Augmented Zagreb index: for a molecular structure $\Gamma$, the augmented Zagreb index [13] is

$$
\operatorname{AZI}(\Gamma)=\sum_{s t \in E(\Gamma)}\left[\frac{\varphi(s) \times \varphi(t)}{\varphi(s)+\varphi(t)-2}\right]^{3} .
$$

A graph polynomial is a graph invariant whose values are polynomials. So, all these invariants are discussed in algebraic graph theory [36]. Among such types of algebraic polynomials, the M-polynomial, defined in 2015, shows the same role in finding the much closed form of various degree-based TIs that correlate different types of chemical properties of the various materials under 
investigation. In 2019, Yang et al. [37] find out the M-polynomial and topological indices of benzene ring embedded in P-type surface network. In 2020, Khalaf et al. [38] computed the M-polynomial and topological indices of book graph and Raza and Sakaiti [2] solved the M-polynomial and degree-based topological indices of some nanostructures. In 2021, Mondal et al. [39] find out the neighborhood M-polynomial of titanium compounds and Irfan et al. [1] computed the M-polynomials and topological indices for line graphs of chain silicate network and $\mathrm{H}$-naphtalenic nanotubes.

M-Polynomial: let $\Gamma$ be a molecular structure and $m_{i, j} \Gamma, i, j \geq 1$, be the number of edges $e=s t$ of $\Gamma$ in such a way that $\{\varphi(s) \varphi(t)\}=\{i, j\}$. The M-polynomial of $\Gamma$ is

$$
M(\Gamma, \mu, \nu)=\sum_{i \leq j(\Gamma)}\left(m_{i, j} \Gamma \mu^{i} \nu^{j}\right) .
$$

Now, we discussed the relationship between the M-polynomial and some important TIs in the form of Tables 1 and 2.

\section{Pent-Heptagonal Nanosheet}

Firstly, we discuss the structure of pent-heptagonal nanosheet $V C_{5} C_{7}$. For nanosheet of $V C_{5} C_{7}(a, b)$, we represent the number of pentagons in the first row by $b$, and the first four rows of nodes as well as edges are repeated. Therefore, we represent the number of repetitions as $a$. The nanosheet $V C_{5} C_{7}(2,4)$ has $16 a b+2 a+5 b$ nodes or vertices and $24 a b+4 b$ edges. Additionally, it has $6 a+7 b$ nodes having degree 2 and $16 a b-4 a-2 b$ nodes having degree 3 . The degree-based edge partition of nanosheet $a=2$ and $b=4$ is shown in Table 3.

From Figure 1, we note that 2 distinct types of vertices in $V C_{5} C_{7}$ are 2 and 3. So,

$$
\begin{gathered}
V_{1}=\left\{s \in V\left(\Gamma_{1}\right) \mid \varphi(s)=2\right\} \\
V_{2}=\left\{s \in V\left(\Gamma_{1}\right) \mid \varphi(s)=3\right\} .
\end{gathered}
$$

We have 3 different types of edges that is based on the degree of end nodes in $\left(\Gamma_{1}\right)$ that are

$$
\begin{aligned}
& E_{2,2}=\left\{s t \in\left(\Gamma_{1}\right) \mid \varphi(s)=2, \varphi(t)=2\right\} \\
& E_{2,3}=\left\{s t \in\left(\Gamma_{1}\right) \mid \varphi(s)=2, \varphi(t)=3\right\} \\
& E_{3,3}=\left\{s t \in\left(\Gamma_{1}\right) \mid \varphi(s)=3, \varphi(t)=3\right\},
\end{aligned}
$$

where $\quad\left|E_{1}\right|=(2 a+2 b+4), \quad\left|E_{2}\right|=(8 a+10 b-8)$, $\left|E_{3}\right|=(24 a b-10 a-8 b+4)$, and $a=2$ and $b=4$. Then,

$$
\left|E\left(\Gamma_{1}\right)\right|=\left|E_{1}\right|+\left|E_{2}\right|+\left|E_{3}\right|=16+48+144=208 .
$$

Now, we discuss the structure of pent-heptagonal nanosheet $\mathrm{HC}_{5} \mathrm{C}_{7}$. For the nanosheet $\mathrm{HC}_{5} \mathrm{C}_{7}(a, b)$, we represent the number of pentagons in the first row by $b$, and the 1 st four rows of nodes and edges are repeated. So, we represent the number of repetitions as $a$. The nanosheets $H C_{5} C_{7}(2,4)$ have $16 a b+2 a+4 b$ vertices and $24 a b+3 b$ edges. Moreover, it has $6 a+6 b$ vertices with degree 2 and $16 a b-4 a-2 b$ vertices with degree 3 . The degree-based edge
TABLE 1: Derivation of TIs from M-polynomial.

\begin{tabular}{lcc}
\hline Indices & $f(\mu, \nu)$ & Derivation from $M(\Gamma, \mu, \nu)$ \\
\hline$M_{1}$ & $\mu+\nu$ & $\left.\left(D_{\mu}+D_{\nu}\right)(M(\Gamma, \mu, \nu))\right|_{\mu=1=\nu}$ \\
$M_{2}$ & $\mu \nu$ & $\left.\left(D_{\mu} D_{\nu}\right)(M(\Gamma, \mu, \nu))\right|_{\mu=1=\nu}$ \\
$M M_{2}$ & $1 / \mu \nu$ & $\left.\left(S_{\mu} S_{\nu}\right)(M(\Gamma, \mu, \nu))\right|_{\mu=1=\nu}$ \\
$R_{\alpha}$ & $(\mu \nu)^{\alpha}, \alpha \in N$ & $\left.\left(D_{\mu}^{\alpha} D_{\nu}^{\alpha}\right)(M(\Gamma, \mu, \nu))\right|_{\mu=1=\nu}$ \\
$R_{\alpha} R_{\alpha}$ & $1 /(\mu \nu)^{\alpha}, \alpha \in N$ & $\left.\left(S_{\mu}^{\alpha} S_{\nu}^{\alpha}\right)(M(\Gamma, \mu, \nu))\right|_{\mu=1=\nu}$ \\
$\operatorname{SDD}$ & $\mu^{2}+\nu^{2} / \mu \nu$ & $\left.\left(D_{\mu} S_{\nu}+D_{\nu} S_{\mu}\right)(M(\Gamma, \mu, \nu))\right|_{\mu=1=\nu}$ \\
\hline
\end{tabular}

TABle 2: Other TIs from M-polynomial.

\begin{tabular}{lcc}
\hline Indices & $f(\mu, \nu)$ & Derivation from $M(\Gamma, \mu, \nu)$ \\
\hline$H$ & $2 / \mu+\nu$ & $\left.2 S_{\mu} J(M(\Gamma, \mu, \nu))\right|_{\mu=1=\nu}$ \\
$I S$ & $\mu \nu / \mu+\nu$ & $\left.S_{\mu} Q_{2} J D_{\mu} D_{\nu}(M(\Gamma, \mu, \nu))\right|_{\mu=1=\nu}$ \\
$A Z I$ & $(\mu \nu / \mu+\nu-2)^{3}$ & $\left.S_{\mu}^{3} J D_{\mu}^{3} D_{\nu}^{3}(M(\Gamma, \mu, \nu))\right|_{\mu=1=\nu}$ \\
\hline
\end{tabular}

Table 3: Partition of edge set, $V C_{5} C_{7}$.

\begin{tabular}{lccc}
\hline Edges partitions & $E_{1}=E_{2,2}$ & $E_{2}=E_{2,3}$ & $E_{3}=E_{3,3}$ \\
\hline Cardinality & $2 a+2 b+4$ & $8 a+10 b-8$ & $24 a b-10 a-8 b+4$ \\
\hline
\end{tabular}

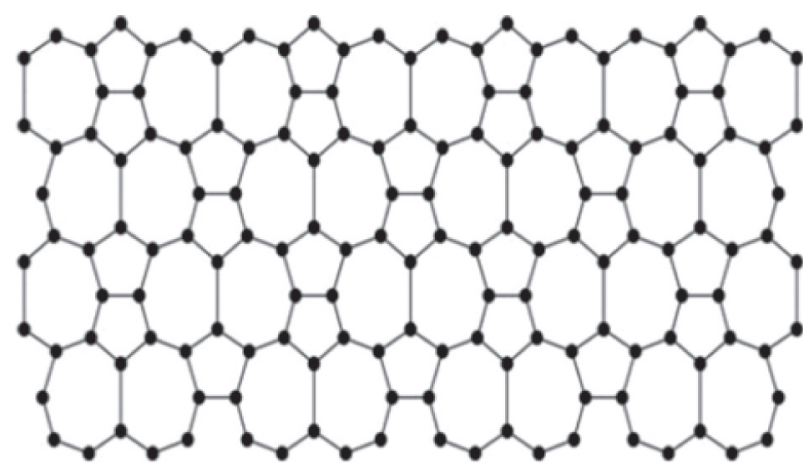

Figure 1: Pent-heptagonal nanosheet $V C_{5} C_{7}$.

partition of nanosheets for $a=2$ and $b=4$ is shown in Table 4.

From Figure 2, we note that 2 distinct types of vertices in $\mathrm{HC}_{5} \mathrm{C}_{7}$ are 2 and 3. So,

$$
\begin{aligned}
& V_{1}=\left\{s \in V\left(\Gamma_{2}\right) \mid \varphi(u)=2\right\}, \\
& V_{2}=\left\{s \in V\left(\Gamma_{2}\right) \mid \varphi(u)=3\right\} .
\end{aligned}
$$

We have 3 different types of edges that is based on the degree of end nodes in $\left(\Gamma_{1}\right)$ :

$$
\begin{aligned}
& E_{2,2}=\left\{s t \in\left(\Gamma_{2}\right) \mid \varphi(s)=2, \varphi(t)=2\right\}, \\
& E_{2,3}=\left\{s t \in\left(\Gamma_{2}\right) \mid \varphi(s)=2, \varphi(t)=3\right\}, \\
& E_{3,3}=\left\{s t \in\left(\Gamma_{2}\right) \mid \varphi(s)=3, \varphi(t)=3\right\} .
\end{aligned}
$$

\section{Main Results}

This section deals with the main results consisting of polynomials and TIs of the nanosheets. 
TABle 4: Partition of edge set of $H C_{5} C_{7}$.

\begin{tabular}{lccc}
\hline Edges' partitions & $E_{1}=E_{2,2}$ & $E_{2}=E_{2,3}$ & $E_{3}=E_{3,3}$ \\
\hline Cardinality & $2 a+3 b+2$ & $8 a+6 b-4$ & $24 a b-10 a-6 b+10$ \\
\hline
\end{tabular}

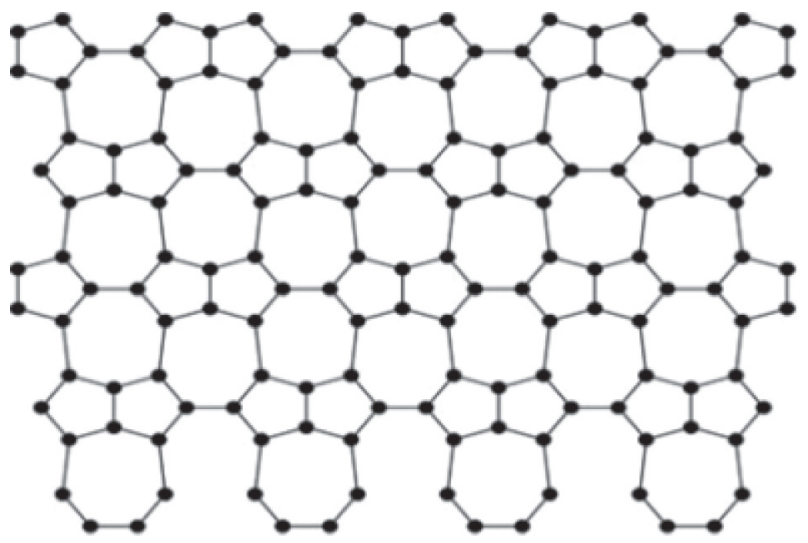

Figure 2: Pent-heptagonal nanosheet $\mathrm{HC}_{5} \mathrm{C}_{7}$.

Theorem 1. Let $\Gamma_{1}=V C_{5} C_{7}$ be the pent-heptagonal nanosheet. Then, the M-polynomial of $\Gamma$ is

$$
\begin{aligned}
M\left(\Gamma_{1}, \mu, \nu\right)= & (2 a+2 b+4) \mu^{2} \nu^{2}+(8 a+10 b-8) \mu^{2} v^{3} \\
& +(24 a b-10 a-8 b+4) \mu^{3} v^{3}
\end{aligned}
$$

Proof. Now, by using definition of M-polynomial of $\left(\Gamma_{1}\right)$, we obtain

$$
\begin{aligned}
M\left(\Gamma_{1}, \mu, \nu\right)= & \sum_{s \leq t}\left[E_{s, t}\left(\Gamma_{1}\right) \mu^{s} v^{t}\right] \\
= & \sum_{2 \leq 2}\left[E_{2,2}\left(\Gamma_{1}\right) \mu^{2} v^{2}\right]+\sum_{2 \leq 3}\left[E_{2,3}\left(\Gamma_{1}\right) \mu^{2} v^{3}\right] \\
& +\sum_{3 \leq 3}\left[E_{3,3}\left(\Gamma_{1}\right) \mu^{3} v^{3}\right] \\
= & \left|E_{1}\right| \mu^{2} v^{2}+\left|E_{2}\right| \mu^{2} v^{3}+\left|E_{3}\right| \mu^{3} v^{3} \\
= & (2 a+2 b+4) \mu^{2} v^{2}+(8 a+10 b-8) \mu^{2} v^{3} \\
& +(24 a b-10 a-8 b+4) \mu^{3} v^{3} .
\end{aligned}
$$

The M-polynomial of $\left(\Gamma_{1}\right)$ is

$$
\begin{aligned}
M\left(\Gamma_{1}, \mu, v\right)= & (2 a+2 b+4) \mu^{2} v^{2}+(8 a+10 b-8) \mu^{2} v^{3} \\
& +(24 a b-10 a-8 b+4) \mu^{3} v^{3}
\end{aligned}
$$

Theorem 2. Let $\Gamma=V C_{5} C_{7}$ be the pent-heptagonal nanosheet. Then, the M-polynomial of $\Gamma$ is

$$
\begin{aligned}
M\left(\Gamma_{1}, \mu, \nu\right)= & (2 a+2 b+4) \mu^{2} \nu^{2}+(8 a+10 b-8) \mu^{2} \nu^{3} \\
& +(24 a b-10 a-8 b+4) \mu^{3} \nu^{3}
\end{aligned}
$$

So, the 1st Zagreb index $\left(M_{1}\left(\Gamma_{1}\right)\right)$, 2nd Zagreb index $\left(M_{2}\left(\Gamma_{1}\right)\right)$, 2nd modified Zagreb $\left(M M_{2}\left(\Gamma_{1}\right)\right)$, general Randic $\left(R_{\gamma}\left(\Gamma_{1}\right)\right)$, reciprocal general Randic $R R_{\gamma}\left(\Gamma_{1}\right)$, where $\gamma \in \alpha$, and the symmetric division deg index $\left(S D D\left(\Gamma_{1}\right)\right)$ obtained from M-polynomial are as follows:
(a) $M_{1}\left(\Gamma_{1}\right)=144 a b-12 a+10 b$
(b) $M_{2}\left(\Gamma_{1}\right)=216 a b-34 a-4 b+4$
(c) $M M_{2}\left(\Gamma_{1}\right)=8 / 3 a b+13 / 18 a+23 / 18 b+1 / 9$
(d) $R_{\gamma}\left(\Gamma_{1}\right)=(4)^{\gamma}(2 a+2 b+4)+(6)^{\gamma}(8 a+10 b-$

$8)+(9)^{\gamma}(24 a b-10 a-8 b+4)$

(e) $R R_{\gamma}\left(\Gamma_{1}\right)=2 a+2 b+4 /(4)^{\gamma}+8 a+10 b-8 /(6)^{\gamma}+$ $24 a b-10 a-8 b+4 /(9)^{\gamma}$

(f) $\operatorname{SSD}\left(\Gamma_{1}\right)=48 a b+4 / 3 a+29 / 3 b-4 / 3$

Proof. Let $f(\mu, \nu)=M\left(\Gamma_{1}, \mu, \nu\right)$ be the M-polynomial of the pent-heptagonal nanosheet $V C_{5} C_{7}$; then,

$$
\begin{aligned}
f(\mu, v)= & (2 a+2 b+4) \mu^{2} v^{2}+(8 a+10 b-8) \mu^{2} v^{3} \\
& +(24 a b-10 a-8 b+4) \mu^{3} v^{3}
\end{aligned}
$$

Firstly, we find out the required partial derivatives and integrals as

$D_{\mu} f(\mu, v)=2(2 a+2 b+4) \mu v^{2}+2(8 a+10 b-8) \mu v^{3}+$ $3(24 a b-10 a-8 b+4) \mu^{2} \nu^{3}$

$D_{\nu} f(\mu, v)=2(2 a+2 b+4) \mu^{2} v+3(8 a+10 b-8) \mu^{2} v^{2}+$ $3(24 a b-10 a-8 b+4) \mu^{3} v^{2}$

$D_{\mu}\left(D_{\nu} f(\mu, \nu)\right)=4(2 a+2 b+4) \mu \nu+6(8 a+10 b-8)$

$\mu \nu^{2}+9(24 a b-10 a-8 b+4) \mu^{2} \nu^{2}$

$T_{\mu}(f(\mu, v))=(a+b+c) \mu^{2} v^{2}+(4 a+5 b-4) \mu^{2} v^{3}+$

$\left(8 a b-10 / 3 a-8 / 3 b+4 / 3 \mu^{3} v^{3}\right)$

$T_{\nu}(f(\mu, v))=(a+b+c) \mu^{2} v^{2}+(8 a+10 b-8) / 3$

$\mu^{2} v^{3}+(8 a b-10 / 3 a-8 / 3 b+4 / 3) \mu^{3} v^{3}$

$T_{\mu} T_{\nu}(f(\mu, \nu))=T_{\mu}\left(T_{\nu}(f(\mu, \nu))\right)=(a+b+2) / 2$

$\mu^{2} v^{2}+(8 a+10 b-8) / 6 \mu^{2} v^{3}+(4 a b-5 / 3 a-4 / 3 b+$

$2 / 3) \mu^{3} v^{3}$

$D_{\nu} T_{\mu}(f(\mu, \nu))=D_{\nu}\left(T_{\mu}(f(\mu, \nu))\right)=2(a+b+c)$ $\mu^{2} v+3(4 a+5 b-4) \mu^{2} v^{2}+3(8 a b-10 / 3 a-8 / 3 b+$ $\left.4 / 3 \mu^{3} v^{2}\right)$

$D_{\mu} T_{\nu}(f(\mu, v))=D_{\mu}\left(T_{\nu}(f(\mu, v))\right)=2(a+b+c)$ $\mu \nu^{2}+2 / 3(8 a+10 b-8) \mu \nu^{3}+(24 a b-10 a-8 b+$ 4) $\mu^{2} v^{3}$

$D_{\mu}^{\gamma} D_{\nu}^{\gamma}=(4)^{\gamma}(2 a+2 b+4) \mu \nu+(6)^{\gamma}(8 a+10 b-8) \mu \nu^{2}+$ $(9)^{\gamma}(24 a b-10 a-8 b+4) \mu^{2} v^{2}$

$T_{\mu}^{\gamma} T_{\nu}^{\gamma}=(2 a+2 b+4) /(4)^{\gamma} \mu^{2} v^{2}+(8 a+10 b-8) /(6)$ $\mu^{2} \nu^{3}+(24 a b-10 a-8 b+4) /(9)^{\gamma} \mu^{3} v^{3}$

Now, we obtain $\mu=\nu=1$ :

$\left.D_{\mu} f(\mu, \nu)\right|_{\mu=v=1}=72 a b-10 a+4$

$\left.D_{\nu} f(\mu, \nu)\right|_{\mu=v=1}=72 a b-2 a+10 b-4$

$\left.D_{\mu}\left(D_{\nu} f(\mu, \nu)\right)\right|_{\mu=\nu=1}=216 a b-34 a-4 b+4$

$\left.T_{\mu}(f(\mu, \nu))\right|_{\mu=\nu=1}=8 a b+5 / 3 a+10 / 3 b-8 / 3$

$\left.T_{\nu}(f(\mu, \nu))\right|_{\mu=\nu=1}=8 a b+1 / 3 a+5 / 3 b-4 / 3$ 


$$
\begin{aligned}
& \left.T_{\mu}\left(T_{\nu}(f(\mu, \nu))\right)\right|_{\mu=\nu=1}=8 / 3 a b+13 / 18 a+23 / 18 b-8 / 9 \\
& \left.D_{\nu}\left(T_{\mu}(f(\mu, \nu))\right)\right|_{\mu=\nu=1}=24 a b+4 a+9 b-4 \\
& \left.D_{\mu}\left(T_{\nu}(f(\mu, \nu))\right)\right|_{\mu=v=1}=24 a b-8 / 3 a+2 / 3 b+8 / 3 \\
& \left.D_{\mu}^{\gamma}\left(D_{\nu}^{\gamma}(f(\mu, \nu))\right)\right|_{\mu=\nu=1}=(4)^{\gamma}(2 a+2 b+4)+(6)(8 a+ \\
& 10 b-8)+(9)^{\gamma}(24 a b-10 a-8 b+4) \\
& \left.T_{\mu}^{\gamma}\left(T_{\nu}^{\gamma}(f(\mu, \nu))\right)\right|_{\mu=\nu=1}=(2 a+2 b+4) /(4)^{\gamma}+(8 a+ \\
& 10 b-8) /(6)^{\gamma}+(24 a b-10 a-8 b+4) /(9)^{\gamma}
\end{aligned}
$$

Consequently,

(i) First Zagreb index: $M_{1}\left(\Gamma_{1}\right)=\left(D_{\mu}+D_{\nu}\right)(f(\mu, \nu))$ $\left.\right|_{\mu=v=1}=\left.D_{\mu}(f(\mu, \nu))\right|_{\mu=v=1}+\left.D_{\nu}(f(\mu, \nu))\right|_{\mu=v=1}=(72$ $a b-10 a+4)+(72 a b-2 a+10 b-4)=144 a b-$ $12 a+10 b$

(ii) Second Zagreb index: $M_{2}\left(\Gamma_{1}\right)=\left(D_{\mu} D_{\nu}\right)$ $\left.(f(\mu, \nu))\right|_{\mu=v=1}=\left.D_{\mu}\left(D_{\nu}(f(\mu, \nu))\right)\right|_{\mu=\nu=1}=216 a b-$ $34 a-4 b+4$

(iii) Second modified Zagreb index: $M M_{2}\left(\Gamma_{1}\right)=$ $\left.\left(T_{\mu} T_{\nu}\right)(f(\mu, \nu))\right|_{\mu=v=1}=\left.T_{\mu}\left(T_{\nu}(f(\mu, \nu))\right)\right|_{\mu=v=1}=8 /$ $3 a b+13 / 18 a+23 / 18 b+1 / 9$

(iv) General Randic index: $R_{\gamma}\left(\Gamma_{1}\right)=\left(D_{\mu}^{\gamma} D_{\nu}^{\gamma}\right)(f(\mu, \nu))$ $\left.\right|_{\mu=\gamma=1}=(4)^{\gamma} \quad(2 a+2 b+4)+(6)^{\gamma}(8 a+10 b-8)+$ $(9)^{\gamma}(24 a b-10 a-8 b+4)$

(v) Reciprocal general Randic index: $R R_{\gamma}\left(\Gamma_{1}\right)=(2 a+$ $2 b+4) /(4)^{\gamma} \mu^{2} v^{2}+(8 a+10 b-8) /(6)^{\gamma} \mu^{2} v^{3}+(24 a b$ $-10 a-8 b+4) /(9)^{\gamma}$

(vi) Symmetric division deg index: $\operatorname{SDD}\left(\Gamma_{1}\right)=\left(D_{\mu} T_{\nu}+\right.$ $\left.D_{\nu} T_{\mu}\right)\left.\quad(f(\mu, v))\right|_{\mu=\nu=1}=\left.D_{\mu} T_{\nu}(f(\mu, \nu))\right|_{\mu=v=1}+D_{\nu}$ $\left.T_{\mu}\right)\left.(f(\mu, \nu))\right|_{\mu=\nu=1}=(24 a b-8 / 3+2 / 3 b+8 / 3)+(24$ $a b+4 a+9 b-4)=48 a b+4 / 3 a+29 / 3 b-4 / 3$

Theorem 3. Let $\Gamma_{1}=V C_{5} C_{7}$ be the pent-heptagonal nanosheets. Then, the M-polynomial of $\Gamma_{1}$ is

$$
\begin{aligned}
M(\Gamma, \mu, \nu)= & (2 a+2 b+4) \mu^{2} \nu^{2}+(8 a+10 b-8) \mu^{2} \nu^{3} \\
& +(24 a b-10 a-8 b+4) \mu^{3} v^{3} .
\end{aligned}
$$

Then, harmonic index $\left(H\left(\Gamma_{1}\right)\right)$, inverse index $\left(I S\left(\Gamma_{1}\right)\right)$ and augmented Zagreb index $\left(A Z I\left(\Gamma_{1}\right)\right)$ obtained from M-polynomial are as follows:
(a) $H\left(\Gamma_{1}\right)=13 / 15 a+7 / 3 b+8 a b+2 / 15$
(b) $I S\left(\Gamma_{1}\right)=36 a b-17 / 5 a-44 b+2 / 5$
(c) $A Z I\left(\Gamma_{1}\right)=273.375 a b-33.90625 a+4.875 b+$ 13.5625

Proof. Let $f(\mu, \nu)=M\left(\Gamma_{1}, \mu, \nu\right)$ be the M-polynomial of the pent-heptagonal nanosheets $V C_{5} C_{7}$; then,

$$
\begin{aligned}
f(\mu, \nu)= & (2 a+2 b+4) \mu^{2} \nu^{2}+(8 a+10 b-8) \mu^{2} \nu^{3} \\
& +(24 a b-10 a-8 b+4) \mu^{3} \nu^{3} .
\end{aligned}
$$

Firstly, we find out the required partial derivatives and integrals are as follows:

$$
\begin{aligned}
& J(f(\mu, v))=(2 a+2 b+4) \mu^{4}+(8 a+10 b-8) \mu^{5}+ \\
& (24 a b-10 a-8 b+4) \mu^{6} \\
& T_{\mu}(J(f(\mu, v)))=(a / 2+b / 2+1) \mu^{4}+(8 / 5 a+2 b-8 / 5) \\
& \mu^{5}+(4 a b-5 / 3 a-4 / 3 b+2 / 3) \mu^{6} \\
& J\left(D_{\mu}\left(D_{\nu}(f(\mu, v))\right)\right)=(8 a+8 b+16) \mu^{2}+(48 a+ \\
& 60 b-48) \mu^{3}+(216 a b-90 a-72 b+36) \mu^{4} \\
& Q_{2}\left(J\left(D_{\mu}\left(D_{\nu}(f(\mu, v))\right)\right)\right)=(8 a+8 b+16) \mu^{4}+(48 a+ \\
& 60 b-48) \mu^{5}+(216 a b-90 a-72 b+36) \mu^{6} \\
& T_{\mu}\left(Q_{2}\left(J\left(D_{\mu}\left(D_{\nu}(f(\mu, v))\right)\right)\right)=(2 a+2 b+4) \mu^{4}+1 / 5\right. \\
& (48 a+60 b-48) \mu^{5}+(36 a b-15 a-12 b+6) \mu^{6} \\
& D_{\mu}^{3}\left(D_{\nu}^{3}(f(\mu, v))\right)=(4)^{3}(2 a+2 b+4) \mu \nu+(6)^{3}(8 a+ \\
& 10 b-8) \mu v^{2}+(9)^{3}(24 a b-10 a-8 b+4) \mu^{2} v^{2} \\
& J\left(D_{\mu}^{3} D_{\nu}^{3}(f(\mu, v))\right)=(4)^{3}(2 a+2 b+4) \mu^{2}+(6)^{3}(8 a+ \\
& 10 b-8) \mu^{3}+(9)^{3}(24 a b-10 a-8 b+4) \mu^{4} \\
& T_{\mu}^{3}\left(J\left(D_{\mu}^{3} D_{\nu}^{3}(f(\mu, v))\right)=\mid(4)^{3}(2 a+2 b+4) / 2 \mu^{2}+(6)^{3}\right. \\
& (8 a+10 b-8) / 3 \mu^{3}+(9)^{3}(24 a b-10 a-8 b+4) / 4 \mu^{4}
\end{aligned}
$$

Now, we obtain $\mu=\nu=1$ :

$$
\begin{aligned}
\left.T_{\mu}(J(f(\mu, \nu)))\right|_{\mu=\nu=1}= & \frac{13}{30} a+\frac{7}{6} b+4 a b+\frac{1}{15} \\
\left.T_{\mu}\left(Q_{2}\left(J\left(D_{\mu}\left(D_{\nu}(f(\mu, \nu))\right)\right)\right)\right)\right|_{\mu=\nu=1}= & 36 a b-\frac{17}{5} a+2 b+\frac{2}{5} \\
\left.T_{\mu}^{3}\left(J\left(D_{\mu}^{3} D_{\nu}^{3}(f(\mu, \nu))\right)\right)\right|_{\mu=\nu=1}= & 8(2 a+2 b+4)+8(8 a+10 b-8) \\
& +\left(\frac{729}{64}\right)(24 a b-10 a-8 b+4) \\
= & 273.375 a b-33.90625 a+4.875 b+13.5625 .
\end{aligned}
$$

Consequently,

(i) Harmonic index: 


$$
\begin{aligned}
H\left(\Gamma_{1}\right) & =\left.2 T_{\mu}(J(f(\mu, \nu)))\right|_{\mu=v=1} \\
& =2\left(\frac{13}{30} a+\frac{7}{6} b+4 a b+\frac{1}{15}\right) \\
& =\frac{13}{15} a+\frac{7}{3} b+8 a b+\frac{2}{15}
\end{aligned}
$$

(ii) Inverse index:

$$
\begin{aligned}
\operatorname{IS}\left(\Gamma_{1}\right)= & \left.T_{\mu}\left(Q_{2}\left(J\left(D_{\mu}\left(D_{\nu}(f(\mu, \nu))\right)\right)\right)\right)\right|_{\mu=\nu=1} \\
= & (2 a+2 b+4)+\frac{1}{5}(48 a+60 b-48) \\
& +(36 a b-15 a-12 b+6) \\
= & 36 a b-\frac{17}{5} a+2 b+\frac{2}{5} .
\end{aligned}
$$

(iii) Augmented Zagreb index:

$$
\begin{aligned}
\operatorname{AZI}\left(\Gamma_{1}\right)= & \left.T_{\mu}^{3}\left(J\left(D_{\mu}^{3} D_{\nu}^{3}(f(\mu, \nu))\right)\right)\right|_{\mu=\nu=1} \\
= & \left(\frac{4}{2}\right)^{3}(2 a+2 b+4)+\left(\frac{6}{3}\right)^{3}(8 a+10 b-8) \\
& +\left(\frac{9}{4}\right)^{3}(24 a b-10 a-8 b+4) \\
= & 273.375 a b-33.90625 a+4.875 b+13.5625
\end{aligned}
$$

Theorem 4. Let $\Gamma_{2}=H_{5} C_{7}$ be the second pent-heptagonal nanosheets; the M-polynomial of $\left(\Gamma_{2}\right)$ is

$$
\begin{aligned}
M\left(\Gamma_{2}, \mu, v\right)= & (2 a+3 b+2) \mu^{2} v^{2}+(8 a+6 b-4) \\
& \mu^{2} v^{3}+(24 a b-10 a-6 b+10) \mu^{3} v^{3} .
\end{aligned}
$$

Proof. Now, by using definition of M-polynomial for $\left(\Gamma_{2}\right)$,

$$
\begin{aligned}
M\left(\Gamma_{2}, \mu, \nu\right)= & \sum_{s \leq t}\left[E_{s, t}(\Gamma) \mu^{s} \nu^{t}\right] \\
= & \sum_{2 \leq 2}\left[E_{2,2}\left(\Gamma_{2}\right) \mu^{2} v^{2}\right]+\sum_{2 \leq 3}\left[E_{2,3}\left(\Gamma_{2}\right) \mu^{2} v^{3}\right] \\
& +\sum_{3 \leq 3}\left[E_{3,3}\left(\Gamma_{2}\right) \mu^{3} v^{3}\right] \\
= & \left|E_{1}\right| \mu^{2} v^{2}+\left|E_{2}\right| \mu^{2} v^{3}+\left|E_{3}\right| \mu^{3} v^{3} \\
= & (2 a+3 b+2) \mu^{2} v^{2}+(8 a+6 b-4) \mu^{2} v^{3} \\
& +(24 a b-10 a-6 b+10) \mu^{3} v^{3} .
\end{aligned}
$$

The M-polynomial of $\left(\Gamma_{2}\right)$ is

$$
\begin{aligned}
M\left(\Gamma_{2}, \mu, \nu\right)= & (2 a+3 b+2) \mu^{2} v^{2}+(8 a+6 b-4) \mu^{2} v^{3} \\
& +(24 a b-10 a-6 b+10) \mu^{3} v^{3}
\end{aligned}
$$

Theorem 5. Let $\Gamma_{2}=H C_{5} C_{7}$ be the pent-heptagonal nanosheets. Then, the M-polynomial of $\Gamma_{2}$ is

$$
\begin{aligned}
M\left(\Gamma_{2}, \mu, \nu\right)= & (2 a+2 b+4) \mu^{2} \nu^{2}+(8 a+10 b-8) \mu^{2} v^{3} \\
& +(24 a b-10 a-8 b+4) \mu^{3} v^{3} .
\end{aligned}
$$

So, the 1st Zagreb index $\left(M_{1}\left(\Gamma_{2}\right)\right)$, 2nd modified Zagreb $\left(M M_{2}\left(\Gamma_{2}\right)\right)$, general Randic $\left(R_{\gamma}\left(\Gamma_{2}\right)\right)$ where $\gamma \in \alpha$, reciprocal general Randic $\left(R R_{\gamma}\left(\Gamma_{2}\right)\right)$, where $\gamma \in \alpha$, and the symmetric division deg index $\left(\operatorname{SDD}\left(\Gamma_{2}\right)\right)$ obtained from M-polynomial are as follows:

(a) $M_{1}\left(\Gamma_{2}\right)=144 a b-12 a+6 b+48$

(b) $M_{2}\left(\Gamma_{2}\right)=216 a b-34 a-6 b+74$

(c) $M M_{2}\left(\Gamma_{2}\right)=8 / 3 a b+13 / 18 a+13 / 12 b+17 / 18$

(d) $R_{\gamma}\left(\Gamma_{2}\right)=(4)^{\gamma}(2 a+3 b+2)+(6)^{\gamma}(8 a+6 b-4)+$

$(9)^{\gamma}(24 a b-10 a-6 b+10)$

(e) $R R_{\gamma}\left(\Gamma_{2}\right)=2 a+3 b+2 /(4)^{\gamma}+8 a+6 b-4 /(6)^{\gamma}+$ $24 a b-10 a-6 b+10 /(9)^{\gamma}$

(f) $\operatorname{SSD}\left(\Gamma_{2}\right)=48 a b+4 / 3 a+7 b+46 / 3$

Proof. Let $f(\mu, v)=M\left(\Gamma_{2}, \mu, \nu\right)$ be the M-polynomial of the pent-heptagonal nanosheets $\mathrm{HC}_{5} \mathrm{C}_{7}$; then,

$$
\begin{aligned}
f(\mu, v)= & (2 a+3 b+2) \mu^{2} v^{2}+(8 a+6 b-4) \mu^{2} v^{3} \\
& +(24 a b-10 a-6 b+10) \mu^{3} v^{3} .
\end{aligned}
$$

Firstly, we find out the required partial derivatives and integrals as follows:

$$
\begin{aligned}
& D_{\mu} f(\mu, v)=2(2 a+3 b+2) \mu v^{2}+2(8 a+6 b-4) \mu v^{3}+ \\
& 3(24 a b-10 a-6 b+10) \mu^{2} v^{3} \\
& D_{\nu} f(\mu, \nu)=2(2 a+3 b+2) \mu^{2} v+3(8 a+6 b-4) \mu^{2} v^{2}+ \\
& 3(24 a b-10 a-6 b+10) \mu^{3} v^{2} \\
& D_{\mu}\left(D_{\nu} f(\mu, \nu)\right)=4(2 a+3 b+2) \mu \nu+6(8 a+6 b-4) \\
& \mu \nu^{2}+9(24 a b-10 a-6 b+10) \mu^{2} v^{2} \\
& T_{\mu}(f(\mu, v))=(a+(3 / 2) b+1) \mu^{2} v^{2}+(4 a+3 b-2) \\
& \mu^{2} v^{3}+\left(8 a b-(10 / 3) a-2 b+(10 / 3) \mu^{3} v^{3}\right) \\
& T_{\nu}(f(\mu, v))=(a+(3 / 2) b+1) \mu^{2} v^{2}+((8 / 3) a+2 b- \\
& (4 / 3)) \mu^{2} \nu^{3}+(8 a b-(10 / 3) a-2 b+(10 / 3)) \mu^{3} \nu^{3} \\
& T_{\mu} T_{\nu}(f(\mu, \nu))=T_{\mu}\left(T_{\nu}(f(\mu, \nu))\right)=1 / 4(2 a+3 b+2) \\
& 2 \mu^{2} v^{2}+1 / 6(8 a+6 b-4) \mu^{2} v^{3}+1 / 9(24 a b-10 a-6 b+ \\
& \text { 10) } \mu^{3} v^{3} \\
& D_{\nu} T_{\mu}(f(\mu, \nu))=D_{\nu}\left(T_{\mu}(f(\mu, \nu))\right)=(2 a+3 b+2) \\
& \mu^{2} v+3(4 a+3 b-2) \mu^{2} v^{2}+3(8 a b-10 / 3 a-2 b+ \\
& \left.10 / 3 \mu^{3} v^{2}\right) \\
& D_{\mu} T_{\nu}(f(\mu, \nu))=D_{\mu}\left(T_{\nu}(f(\mu, \nu))\right)=(2 a+3 b+2) \\
& \mu \nu^{2}+2(8 / 3 a+2 b-4 / 3) \mu \nu^{3}+(24 a b-10 a-6 b+10) \\
& \mu^{2} v^{3}
\end{aligned}
$$


$D_{\mu}^{\gamma} D_{\nu}^{\gamma}=(4)^{\gamma}(2 a+3 b+2) \mu \nu+(6)^{\gamma}(8 a+6 b-4) \mu \nu^{2}+$ (9) ${ }^{\gamma}(24 a b-10 a-6 b+10) \mu^{2} v^{2}$

$T_{\mu}^{\gamma} T_{\nu}^{\gamma}=(2 a+3 b+2) /(4)^{\gamma} \mu^{2} v^{2}+(8 a+6 b-4) /(6)^{\gamma}$ $\mu^{2} v^{3}+(24 a b-10 a-6 b+10) /(9)^{\gamma} \mu^{3} v^{3}$

Now, we obtain $\mu=\nu=1$ :

$$
\begin{aligned}
& \left.D_{\mu} f(\mu, v)\right|_{\mu=v=1}=72 a b-10 a+26 \\
& \left.D_{\nu} f(\mu, v)\right|_{\mu=\nu=1}=72 a b-2 a+6 b+22 \\
& \left.D_{\mu}\left(D_{\nu} f(\mu, \nu)\right)\right|_{\mu=v=1}=216 a b-34 a-6 b+74 \\
& \left.T_{\mu}(f(\mu, \nu))\right|_{\mu=\nu=1}=8 a b+5 / 3 a+5 / 2 b+7 / 3 \\
& \left.T_{\nu}(f(\mu, v))\right|_{\mu=v=1}=8 a b+1 / 3 a+3 / 2 b+3 \\
& \left.T_{\mu}\left(T_{\nu}(f(\mu, \nu))\right)\right|_{\mu=\nu=1}=8 / 3 a b+13 / 18 a+13 / 12 b+
\end{aligned}
$$

Consequently,

(i) First Zagreb index: $M_{1}\left(\Gamma_{2}\right)=\left(D_{\mu}+D_{\nu}\right)(f(\mu, \nu))$ $\left.\right|_{\mu=v=1}=\left.D_{\mu}(f(\mu, \nu))\right|_{\mu=v=1}+\left.D_{\nu} \quad(f(\mu, \nu))\right|_{\mu=v=1}=$ $144 a b-12 a+6 b+48$

(ii) Second Zagreb index: $M_{2}\left(\Gamma_{2}\right)=\left(D_{\mu} D_{\nu}\right)(f(\mu, v))$ $\left.\right|_{\mu=v=1}=\left.D_{\mu}\left(D_{\nu}(f(\mu, \nu))\right)\right|_{\mu=v=1}=216 a b-34 a-6 b$ $+74$

(iii) Second modified Zagreb index: $M M_{2}\left(\Gamma_{2}\right)=\left(T_{\mu} T_{\nu}\right)$ $\left.(f(\mu, \nu))\right|_{\mu=\nu=1}=\left.T_{\mu}\left(T_{\nu}(f(\mu, \nu))\right)\right|_{\mu=\nu=1}=8 / 3 a b+$ $13 / 18 a+13 / 12 b+17 / 18$

(iv) General Randic index: $R_{\gamma}\left(\Gamma_{2}\right)=\left(D_{\mu}^{\gamma} D_{\nu}^{\gamma}\right)(f(\mu, \nu))$ $\left.\right|_{\mu=v=1}=(4)^{\gamma}(2 a+3 b+2)+(6)^{\gamma} \quad(8 a+6 b-4)+$ $(9)^{\gamma}(24 a b-10 a-6 b+10)$

(v) Reciprocal general Randic index: $R R_{\gamma}\left(\Gamma_{2}\right)=\left(T_{\mu}^{\gamma} T_{\nu}^{\gamma}\right)$ $\left.(f \quad(\mu, v))\right|_{\mu=v=1}=(2 a+3 b+2) /(4)^{\gamma}+(8 a+6 b-$ $4) /(6)^{\gamma}+(24 a b-10 a-6 b+10) /(9)^{\gamma}$

(vi) Symmetric division deg index: $\operatorname{SDD}\left(\Gamma_{2}\right)=\left(D_{\mu} T_{\nu}+\right.$ $\left.D_{\nu} T_{\mu}\right)\left.(f(\mu, \nu)) \quad\right|_{\mu=v=1}=\left.D_{\mu} T_{\nu}(f(\mu, \nu))\right|_{\mu=v=1}+D_{\nu}$ $\left.T_{\mu}\right)\left.(f(\mu, v))\right|_{\mu=v=1}=(24 a b-8 / 3 a+b+28 / 3)+(24$ $a b+4 a+6 b+6)=48 a b+4 / 3 a+7 b+46 / 3$
Theorem 6. Let $\Gamma_{2}=H C_{5} C_{7}$ be the pent-heptagonal nanosheets. Then, the M-polynomial of $\Gamma_{2}$ is

$$
\begin{aligned}
M\left(\Gamma_{2}, \mu, \nu\right)= & (2 a+3 b+2) \mu^{2} \nu^{2}+(8 a+6 b-4) \mu^{2} \nu^{3} \\
& +(24 a b-10 a-6 b+10) \mu^{3} v^{3} .
\end{aligned}
$$

Then, harmonic index $\left(H\left(\Gamma_{2}\right)\right)$, inverse index $\left(\operatorname{IS}\left(\Gamma_{2}\right)\right)$, and augmented Zagreb index $\left(\mathrm{AZI}\left(\Gamma_{2}\right)\right)$ obtained from M-polynomial are as follows:

(a) $H\left(\Gamma_{2}\right)=13 / 15 a+19 / 10 b+8 a b-11 / 3$

(b) IS $\left(\Gamma_{2}\right)=36 a b-17 / 5 a-9 / 5 b+61 / 5$

(c) $\operatorname{AZI}\left(\left(\Gamma_{2}\right)=273.3744 a b-33.90625 a+3.6564 b+\right.$ 97.906

Proof. Let $f(\mu, \nu)=M\left(\Gamma_{2}, \mu, \nu\right)$ be the M-polynomial of the pent-heptagonal nanosheet $\mathrm{HC}_{5} \mathrm{C}_{7}$; then,

$$
\begin{aligned}
f(\mu, \nu)= & (2 a+3 b+2) \mu^{2} v^{2}+(8 a+6 b-4) \mu^{2} \nu^{3} \\
& +(24 a b-10 a-6 b+10) \mu^{3} v^{3} .
\end{aligned}
$$

First, we find out the required partial derivatives and integrals as

$$
\begin{aligned}
& J(f(\mu, v))=(2 a+3 b+2) \mu^{4}+(8 a+6 b-4) \mu^{5}+ \\
& (24 a b-10 a-6 b+10) \mu^{6} \\
& T_{\mu}(J(f(\mu, v)))=2 a+3 b+2 / 4 \mu^{4}+8 a+6 b-4 / 5 \mu^{5}+ \\
& 24 a b-10 a-6 b+10 / 6 \mu^{6} \\
& J\left(D_{\mu}\left(D_{\nu}(f(\mu, v))\right)\right)=(8 a+12 b+8) \mu^{2}+(48 a+ \\
& 36 b-24) \mu^{3}+(216 a b-90 a-54 b+90) \mu^{4} \\
& Q_{2}\left(J\left(D_{\mu}\left(D_{\nu}(f(\mu, v))\right)\right)\right)=(8 a+12 b+8) \mu^{4}+(48 a+ \\
& 36 b-24) \mu^{5}+(216 a b-90 a-54 b+90) \mu^{6} \\
& T_{\mu}\left(Q_{2}\left(J\left(D_{\mu}\left(D_{\nu}(f(\mu, v))\right)\right)\right)\right)=(2 a+3 b+2) \mu^{4}+ \\
& 1 / 5(48 a+36 b-24) \mu^{5}+(36 a b-15 a-9 b+15) \mu^{6} \\
& D_{\mu}^{3}\left(D_{\nu}^{3}(f(\mu, v))\right)=(4)^{3}(2 a+3 b+2) \mu \nu+(6)^{3}(8 a+ \\
& 6 b-4) \mu v^{2}+(9)^{3}(24 a b-10 a-6 b+10) \mu^{2} v^{2} \\
& J\left(D_{\mu}^{3} D_{\nu}^{3}(f(\mu, v))\right)=(4)^{3}(2 a+3 b+2) \mu^{2}+(6)^{3}(8 a+ \\
& 6 b-4) \mu^{3}+(9)^{3}(24 a b-10 a-6 b+10) \mu^{4} \\
& T_{\mu}^{3}\left(J\left(D_{\mu}^{3} D_{\nu}^{3}(f(\mu, \nu))\right)\right)=8(2 a+3 b+2) \mu^{2}+8(8 a+ \\
& 6 b-4) \mu^{3}+(9 / 4)^{3}(24 a b-10 a-6 b+10 / 4) \mu^{4}
\end{aligned}
$$

Now, we obtain $\mu=\nu=1$ :

$$
\begin{aligned}
\left.T_{\mu}(J(f(\mu, \nu)))\right|_{\mu=\nu=1} & =\frac{1}{4}(2 a+3 b+2)+\frac{1}{5}(8 a+6 b-4)+\frac{1}{6}(24 a b-10 a-6 b+10) \\
& =\frac{13}{30} a+\frac{19}{20}+4 a b+\frac{41}{30} \\
\left.T_{\mu}\left(Q_{2}\left(J\left(D_{\mu}\left(D_{\nu}(f(\mu, \nu))\right)\right)\right)\right)\right|_{\mu=\nu=1} & =\frac{1}{4}(8 a+12 b+8)+\frac{1}{5}(48 a+36 b-24)+\frac{1}{6}(216 a b-90 a-54 b+90) \\
& =\frac{1}{5}(180 a b-17 a-6 b+36),
\end{aligned}
$$


TABLe 5: Comparison between $M_{1}\left(\Gamma_{1}\right), M_{2}\left(\Gamma_{1}\right), M M_{1}\left(\Gamma_{1}\right)$, and $\operatorname{SDD}\left(\Gamma_{1}\right)$ of $V C_{5} C_{7}$

\begin{tabular}{|c|c|c|c|c|}
\hline$a, b$ & $M_{1}\left(\Gamma_{1}\right)$ & $M_{2}\left(\Gamma_{1}\right)$ & $M M_{1}\left(\Gamma_{1}\right)$ & $\operatorname{SDD}\left(\Gamma_{1}\right)$ \\
\hline$a=2, b=4$ & 1168 & 1648 & 28.016 & 423.91 \\
\hline$a=4, b=6$ & 3468 & 5028 & 74.68 & 1214 \\
\hline$a=6, b=8$ & 6920 & 10134 & 142.69 & 2390.68 \\
\hline$a=8, b=10$ & 11524 & 16972 & 232.02 & 3945.99 \\
\hline$a=10, b=12$ & 17280 & 25536 & 343.086 & 5887.99 \\
\hline$a=12, b=14$ & 24188 & 35828 & 475.248 & 8214 \\
\hline$a=14, b=16$ & 32248 & 47848 & 628.77 & 10924.14 \\
\hline$a=16, b=18$ & 41460 & 61596 & 803.652 & 14018.16 \\
\hline$a=18, b=20$ & 51824 & 77072 & 999.894 & 17496.18 \\
\hline$a=20, b=22$ & 63340 & 94276 & 1217.496 & 21358.2 \\
\hline
\end{tabular}

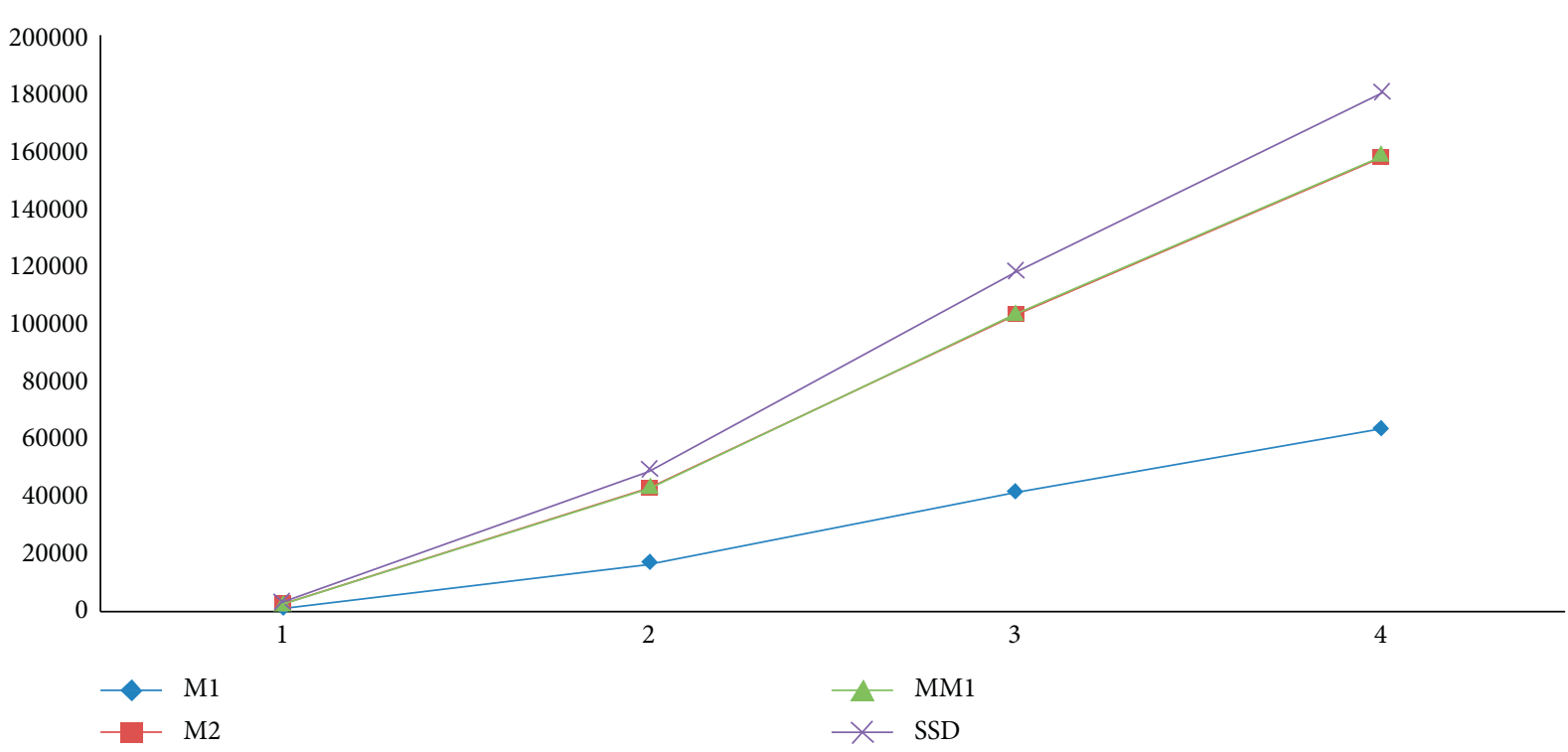

Figure 3: Graphical comparison between $M_{1}\left(\Gamma_{1}\right), M_{2}\left(\Gamma_{1}\right), M M_{1}\left(\Gamma_{1}\right)$, and $\operatorname{SDD}\left(\Gamma_{1}\right)$ of $V C_{5} C_{7}$ and comparison between $M_{1}\left(\Gamma_{2}\right), M_{2}\left(\Gamma_{2}\right)$, $M M_{1}\left(\Gamma_{2}\right)$, and $\operatorname{SDD}\left(\Gamma_{2}\right)$ of $H C_{5} C_{7}$.

TABle 6: Comparison between $M_{1}\left(\Gamma_{2}\right), M_{2}\left(\Gamma_{2}\right), M M_{1}\left(\Gamma_{2}\right)$, and $\operatorname{SDD}\left(\Gamma_{2}\right)$ of $H C_{5} C_{7}$.

\begin{tabular}{|c|c|c|c|c|}
\hline$a, b$ & $M_{1}\left(\Gamma_{2}\right)$ & $M_{2}\left(\Gamma_{2}\right)$ & $M M_{1}\left(\Gamma_{2}\right)$ & $\operatorname{SDD}\left(\Gamma_{2}\right)$ \\
\hline$a=2, b=4$ & 1200 & 1710 & 28.07 & 429.97 \\
\hline$a=4, b=6$ & 3492 & 5086 & 74.33 & 1214.66 \\
\hline$a=6, b=8$ & 6936 & 10190 & 141.94 & 2383.33 \\
\hline$a=8, b=10$ & 11532 & 17022 & 231.105 & 3936.06 \\
\hline$a=10, b=12$ & 17280 & 25582 & 341.505 & 5872.74 \\
\hline$a=12, b=14$ & 24180 & 35870 & 473.265 & 8193.42 \\
\hline$a=14, b=16$ & 32232 & 47886 & 626.385 & 10898.1 \\
\hline$a=16, b=18$ & 41436 & 61630 & 800.865 & 13986.78 \\
\hline$a=18, b=20$ & 51792 & 77102 & 996.705 & 17459.46 \\
\hline$a=20, b=22$ & 63300 & 94302 & 1211.745 & 21316.14 \\
\hline
\end{tabular}

$$
\begin{aligned}
\left.T_{\mu}^{3}\left(J\left(D_{\mu}^{3} D_{\nu}^{3}(f(\mu, v))\right)\right)\right|_{\mu=\nu=1} & =8(2 a+3 b+2)+8(8 a+6 b-4)+\left(\frac{9}{4}\right)^{3}(24 a b-10 a-6 b+10) \\
& =8(2 a+3 b+2)+8(8 a+6 b-4)+(11.3906)(24 a b-10 a-6 b+10) \\
& =273.3744 a b-33.90625 a+3.6564 b+97.906 .
\end{aligned}
$$

Consequently,

(i) Harmonic index: 


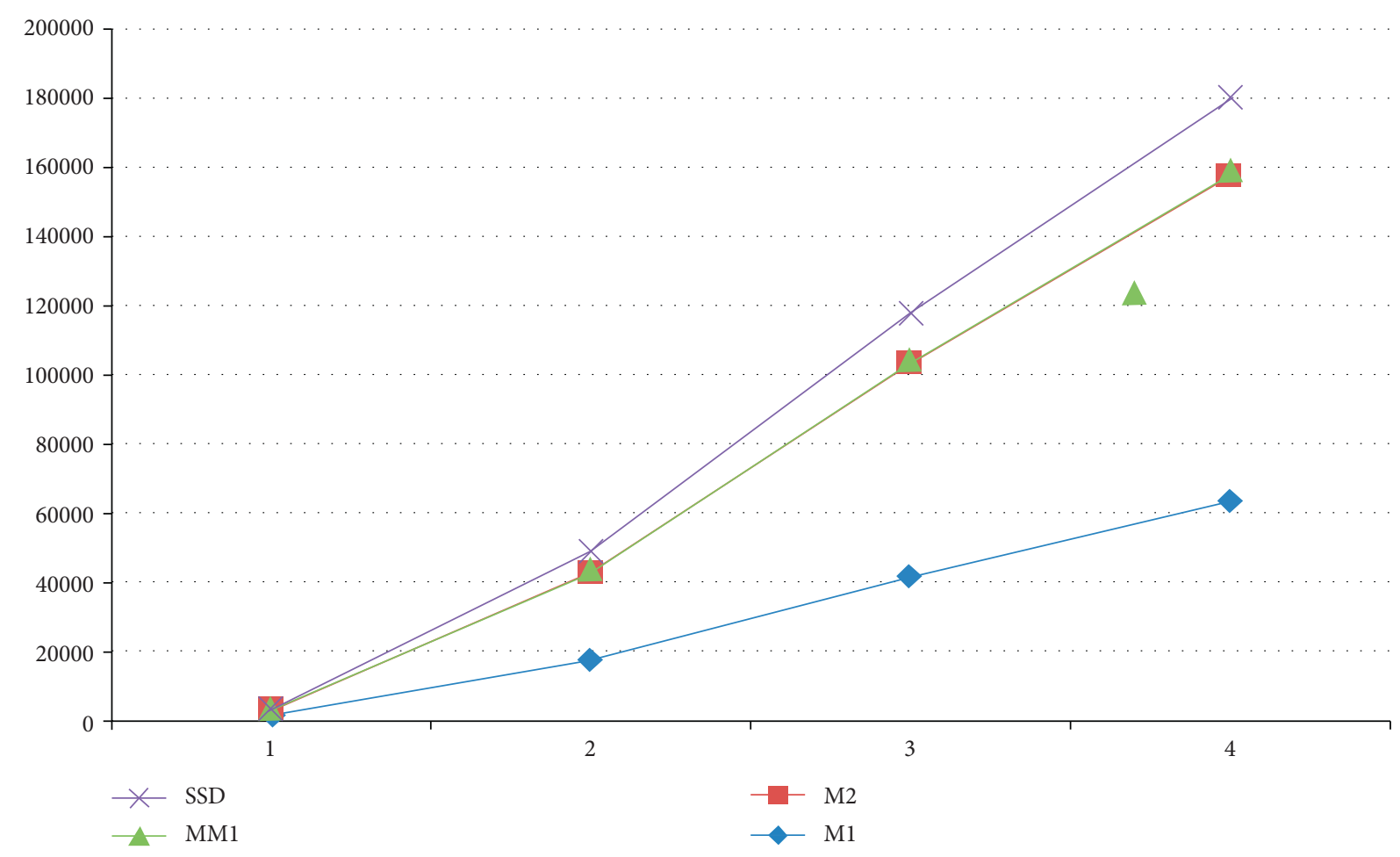

FIGURE 4: Graphical comparison between $M_{1}\left(\Gamma_{2}\right), M_{2}\left(\Gamma_{2}\right), M M_{1}\left(\Gamma_{2}\right)$, and $\operatorname{SDD}\left(\Gamma_{2}\right)$ of $H C_{5} C_{7}$ and comparison between $H\left(\Gamma_{1}\right)$, IS $\left(\Gamma_{1}\right)$, and $\mathrm{AZI}\left(\Gamma_{1}\right)$ of $\mathrm{VC}_{5} \mathrm{C}_{7}$.

TABle 7: Comparison between $H\left(\Gamma_{1}\right)$, IS $\left(\Gamma_{1}\right)$, and AZI $\left(\Gamma_{1}\right)$ of $V C_{5} C_{7}$.

\begin{tabular}{lccc}
\hline$a, b$ & $H\left(\Gamma_{1}\right)$ & IS $\left(\Gamma_{1}\right)$ & AZI $\left(\Gamma_{1}\right)$ \\
\hline$a=2, b=4$ & 75.208 & 105.6 & 2152.25 \\
$a=4, b=6$ & 209.68 & 586 & 6468.18 \\
$a=6, b=8$ & 408 & 1354 & 12971.12 \\
$a=8, b=10$ & 670.454 & 2413.2 & 21661.1125 \\
$a=10, b=12$ & 996.864 & 3758.4 & 32538.0625 \\
$a=12, b=14$ & 1387.274 & 5391.6 & 45602.0125 \\
$a=14, b=16$ & 1841.684 & 7312.8 & 60852.9625 \\
$a=16, b=18$ & 2360.094 & 9522 & 78290.9125 \\
$a=18, b=20$ & 2942.504 & 12019.2 & 97915.8625 \\
$a=20, b=22$ & 3588.914 & 14804.4 & 119727.8125 \\
\hline
\end{tabular}

$$
\begin{aligned}
H\left(\Gamma_{2}\right) & =\left.2 T_{\mu}(J(f(\mu, v)))\right|_{\mu=v=1} \\
& =2\left[\frac{1}{4}(2 a+3 b+2)+\frac{1}{5}(8 a+6 b-4)+\frac{1}{6}(24 a b-10 a-6 b+10)\right] \\
& =8 a b+\frac{13}{15} a+\frac{19}{10} b-\frac{11}{3} .
\end{aligned}
$$

(ii) Inverse index: 


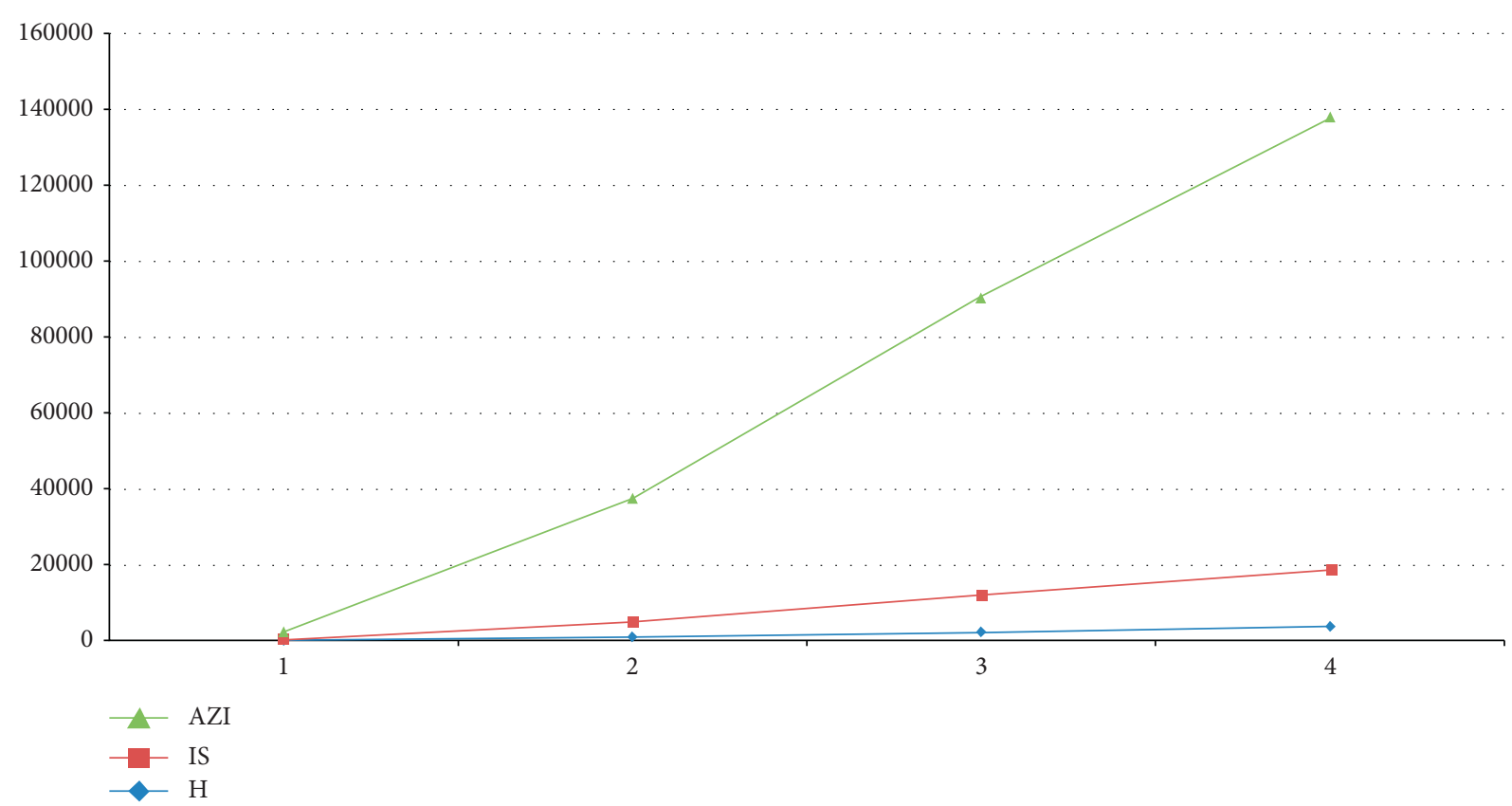

FIGURE 5: Graphical comparison between $H\left(\Gamma_{1}\right)$, IS $\left(\Gamma_{1}\right)$, and $\mathrm{AZI}\left(\Gamma_{1}\right)$ of $V C_{5} C_{7}$ and comparison between $H\left(\Gamma_{2}\right), \operatorname{IS}\left(\Gamma_{2}\right)$, and $\mathrm{AZI}\left(\Gamma_{2}\right)$ of $\mathrm{HC}_{5} \mathrm{C}_{7}$.

TABLE 8: Comparison between $H\left(\Gamma_{2}\right)$, IS $\left(\Gamma_{2}\right)$, and $\mathrm{AZI}\left(\Gamma_{2}\right)$ of $H C_{5} C_{7}$.

\begin{tabular}{lccc}
\hline$a, b$ & $H\left(\Gamma_{2}\right)$ & IS $\left(\Gamma_{2}\right)$ & AZI $\left(\Gamma_{2}\right)$ \\
\hline$a=2, b=4$ & 69.66 & 236.2 & 2231.7143 \\
$a=4, b=6$ & 203.20 & 851.80 & 6545.22 \\
$a=6, b=8$ & 400.73 & 1705.40 & 13045.69 \\
$a=8, b=10$ & 662.266 & 2847 & 21732.8 \\
$a=10, b=12$ & 987.8 & 4276.6 & 32607.1 \\
$a=12, b=14$ & 1377.34 & 599.2 & 45668.36 \\
$a=14, b=16$ & 1830.86 & 8000 & 60916.58 \\
$a=16, b=18$ & 2348.402 & 10293.4 & 78351.76 \\
$a=18, b=20$ & 2929.936 & 12875 & 97974.1 \\
$a=20, b=22$ & 3575.47 & 15744.6 & 119782.2 \\
\hline
\end{tabular}

$$
\begin{aligned}
\operatorname{IS}\left(\Gamma_{2}\right) & =\left.T_{\mu}\left(Q_{2}\left(J\left(D_{\mu}\left(D_{\nu}(f(\mu, \nu))\right)\right)\right)\right)\right|_{\mu=\nu=1} \\
& =\frac{1}{4}(8 a+12 b+8)+\frac{1}{5}(48 a+36 b-24)+\frac{1}{6}(216 a b-90 a-54 b+90) \\
& =36 a b-\frac{17}{5} a-\frac{9}{5} b+\frac{61}{5} .
\end{aligned}
$$

(iii) Augmented Zagreb index: 


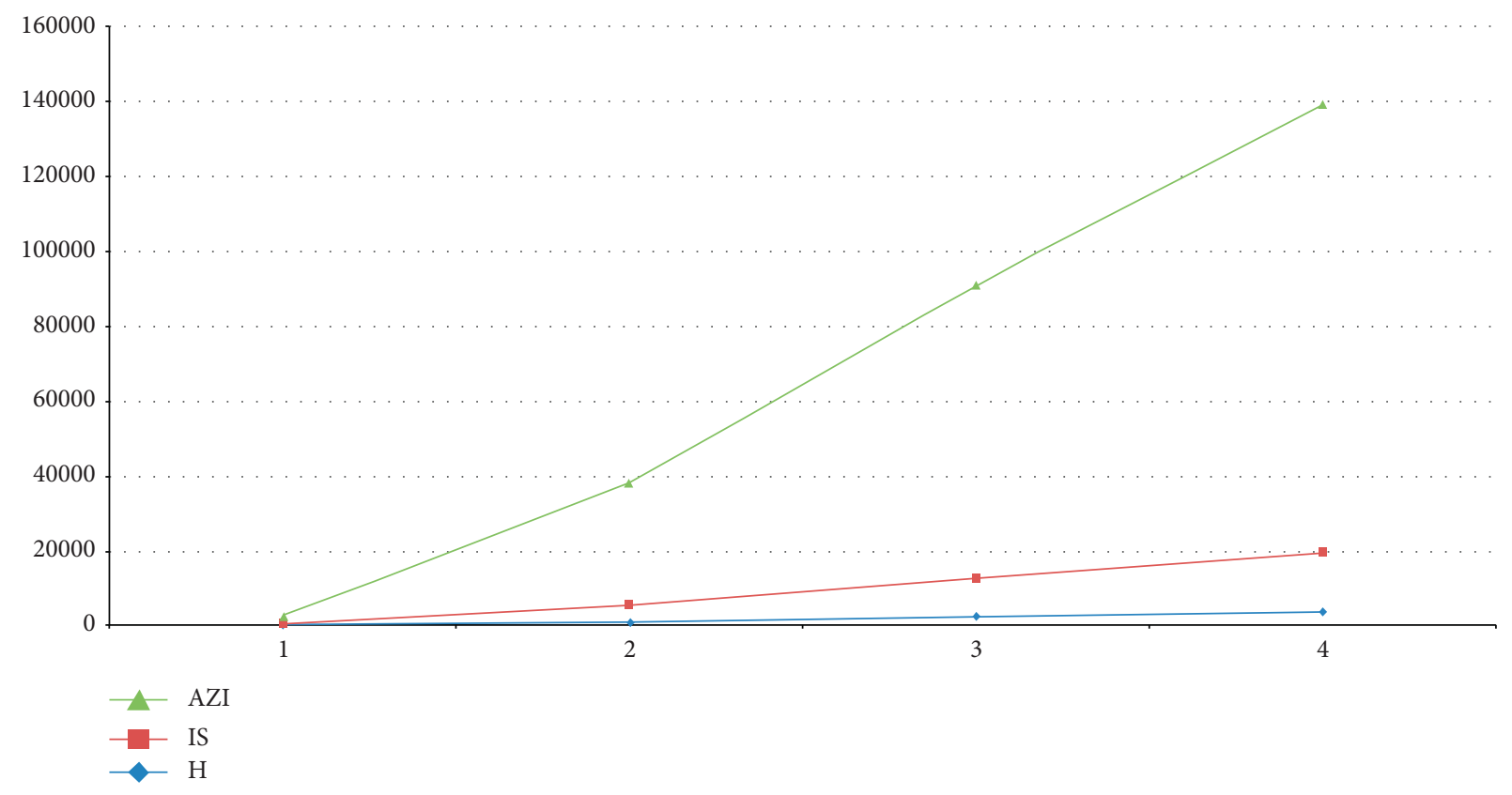

Figure 6: Graphical comparison between $H\left(\Gamma_{2}\right)$, IS $\left(\Gamma_{2}\right)$, and AZI $\left(\Gamma_{2}\right)$ of $H C_{5} C_{7}$.

$$
\begin{aligned}
\operatorname{AZI}\left(\Gamma_{2}\right) & =\left.T_{\mu}^{3}\left(J\left(D_{\mu}^{3} D_{\nu}^{3}(f(\mu, \nu))\right)\right)\right|_{\mu=v=1} \\
& =(2 a+3 b+2)+8(8 a+6 b-4)+\left(\frac{9}{4}\right)^{3}(24 a b-10 a-6 b+10) \\
& =8(2 a+3 b+2)+8(8 a+6 b-4)+(11.3906)(24 a b-10 a-6 b+10) \\
& =273.3744 a b-33.90625 a+3.6564 b+97.906
\end{aligned}
$$

\section{Conclusion}

In this section, we used the various degree-based TIs and show the comparison in the form of tables and figures. Comparison between $M_{1}\left(\Gamma_{1}\right), M_{2}\left(\Gamma_{1}\right), M M_{1}\left(\Gamma_{1}\right)$, and $\operatorname{SDD}\left(\Gamma_{1}\right)$ of $V C_{5} C_{7}$

The comparison of 1st Zagreb, 2nd Zagreb, 2nd modified Zagreb, and symmetric division deg indices of pent-heptagonal nanosheets $\left(\Gamma_{1}\right)$ is computationally computed by using these M-polynomials. We calculated these indices for different values of $a$ and $b$ in Table 5 , and we noted that when we increase the values of $a$ and $b$, then all of the TIs of $V_{5} C_{7}$ are increasing with the same order, as shown in Figure 3.

The comparison of 1st Zagreb, 2nd Zagreb, 2nd modified Zagreb, and symmetric division deg indices of pent-heptagonal nanosheets $\left(\Gamma_{2}\right)$ is computationally computed by using these M-polynomials. We calculated these indices for different values of $a$ and $b$ in Table 6 , and we noted that when we increase the values of $a$ and $b$, then all of the TIs of $\mathrm{HC}_{5} \mathrm{C}_{7}$ are increasing with the same order, as shown in Figure 4.

The comparison of the harmonic index, the inverse sum index, and the augmented Zagreb index of pent-heptagonal nanosheets $\left(\Gamma_{1}\right)$ is computationally computed by these
M-polynomials. We calculated these indices for different values of $a$ and $b$ in Table 7 , and we noted that when we increase the values of $a$ and $b$, then all of the TIs of $V C_{5} C_{7}$ are increasing with the same order, as shown in Figure 5.

The comparison of the harmonic index, the inverse sum index, and the augmented Zagreb index of pent-heptagonal nanosheets $\left(\Gamma_{2}\right)$ is computationally computed by these M-polynomials. We calculated these indices for different values of $a$ and $b$ in Table 8 , and we noted that when we increase the values of $a$ and $b$, then all of the TIs of $\mathrm{HC}_{5} \mathrm{C}_{7}$ are increasing with the same order, as shown in Figure 6.

In this paper, the calculated M-polynomials and enumerated TIs assist us to recognize the physical characteristic, chemical sensitivity, and biological animation of the pentheptagonal nanosheets $\left(\Gamma_{1}\right)$ and $\left(\Gamma_{2}\right)$. These consequences give us remarkable ascertainment in the field of pharmaceutical production.

However, the problem is still open to compute the different TIs (degree and distance based) for various nanosheets:

(i) To compute the nanosheet for other topological indices

(ii) To compute the various nanosheets for different topological indices 


\section{Data Availability}

No data were used to support this study.

\section{Conflicts of Interest}

The authors declare that there are no conflicts of interest regarding this publication.

\section{References}

[1] M. Irfan, H. U. Rehman, H. Almusawa, S. Rasheed, and I. A. Baloch, "M-polynomials and topological indices for line graphs of chain silicate network and h-naphtalenic nanotubes," Journal of Mathematics, vol. 2021, Article ID 5551825, 11 pages, 2021.

[2] Z. Raza and E. K. Sukaiti, "M-polynomial and degree based topological indices of some nanostructures," Symmetry, vol. 12 , no. 5, p. 831, 2020.

[3] K. Culik, Applications of Graph Theory to Mathematical Logic and Linguistics. Theory of Graphs and it Applications, Czech Academy of Sciences, Prague, Czechia, 1963.

[4] M. Baca, J. Horvthov, M. Mokriov, A. Semanicov-Fenovckov, and A. Suhnyiov, "On topological indices of a carbon nanotube network," Canadian Journal of Chemistry, vol. 93, no. 10, pp. 1157-1160, 2015.

[5] O. M. Yaghi, M. O’Keeffe, N. W. Ockwig, H. K. Chae, M. Eddaoudi, and J. Kim, "A route to high surface area, porosity and inclusion of large molecules in crystals," Nature, vol. 423, no. 6941, pp. 705-714, 2003.

[6] H. Gonzalez-Diaz, S. Vilar, L. Santana, and E. Uriarte, "Medicinal chemistry and bioinformatics-current trends in drugs discovery with networks topological indices," Current Topics in Medicinal Chemistry, vol. 7, no. 10, pp. 1015-1029, 2007.

[7] M. R. Farahani, "Connectivity indices of pent-heptagonal nanotubes," Advance in Materials and Corrosion, vol. 2, pp. 33-35, 2013.

[8] S. Hayat and M. Imran, "Computation of certain topological indices of nanotubes covered by C 5 and C 7," Journal of Computational and Theoretical Nanoscience, vol. 12, no. 4, pp. 533-541, 2015.

[9] S. Klavar and I. Gutman, "A comparison of the schultz molecular topological index with the wiener index," Journal of Chemical Information and Computer Sciences, vol. 36, no. 5, pp. 1001-1003, 1996.

[10] X. Li and J. Zheng, "Extremal chemical trees with minimum or maximum general randic index," MATCH Communications in Mathematical and in Computer Chemistry, vol. 55, no. 2, pp. 381-390, 2006.

[11] A. W. Bharati Rajan, C. Grigorious, and S. Stephen, "On certain topological indices of silicate, honeycomb and hexagonal networks," Journal of Computer and Mathematical Sciences, vol. 3, no. 5, pp. 498-556, 2012.

[12] A. Graovac, M. Ghorbani, and M. A. Hosseinzadeh, "Computing fifth geometric-arithmetic index for nanostar dendrimers," Journal of Mathematical Nanoscience, vol. 1, no. 1-2, pp. 33-42, 2011.

[13] B. Furtula, A. Graovac, and D. Vukičević, "Augmented zagreb index," Journal of Mathematical Chemistry, vol. 48, no. 2, pp. 370-380, 2010.

[14] K. C. Das and N. Trinajstić, "Relationship between the eccentric connectivity index and zagreb indices," Computers \&
Mathematics with Applications, vol. 62, no. 4, pp. 1758-1764, 2011.

[15] C. K. Gupta, V. Lokesha, S. B. Shwetha, and P. S. Ranjini, "On the symmetric division deg index of graph," Southeast Asian Bulletin of Mathematics, vol. 40, no. 1, 2016.

[16] A. R. Matamala and E. Estrada, "Generalised topological indices: optimisation methodology and physico-chemical interpretation," Chemical Physics Letters, vol. 410, no. 4-6, pp. 343-347, 2005.

[17] A. Rani and U. Ali, "Degree-Based topological indices of polysaccharides: amylose and blue starch-iodine complex," Journal of Chemistry, vol. 2021, Article ID 6652014, 10 pages, 2021.

[18] G. Abbas, A. Rani, M. Salman, T. Noreen, and U. Ali, "Hosoya properties of the commuting graph associated with the group of symmetries," Main Group Metal Chemistry, vol. 44, no. 1, pp. 173-184, 2021.

[19] J. Devillers, D. Domine, C. Guillon, S. Bintein, and W. Karcher, "Prediction of partition coefficients (log p oct) using autocorrelation descriptors," SAR and QSAR in Environmental Research, vol. 7, no. 1-4, pp. 151-172, 1997.

[20] I. Gutman and O. E. Polansky, Mathematical Concepts in Organic Chemistry, Springer Science \& Business Media, Berlin, Germany, 2012.

[21] G. Plya, "Algebraische berechnung der anzahl der isomeren einiger organischer verbindungen," Zeitschrift fr Kristallographie-Crystalline Materials, vol. 93, no. 1, pp. 415-443, 1936.

[22] H. Wiener, "Structural determination of paraffin boiling points," Journal of the American Chemical Society, vol. 69, pp. 17-20, 1947.

[23] S. Akhter and M. Imran, "On molecular topological properties of benzenoid structures," Canadian Journal of Chemistry, vol. 94, no. 8, pp. 687-698, 2016.

[24] M. Baca, J. Horvthov, M. Mokriov, and A. Suhnyiov, "On topological indices of fullerenes," Applied Mathematics and Computation, vol. 251, pp. 154-161, 2015.

[25] F. M. Brckler, T. Dolic, A. Graovac, and I. Gutman, "On a class of distance-based molecular structure descriptors," Chemical Physics Letters, vol. 503, no. 4-6, pp. 336-338, 2011.

[26] B. Furtula and I. Gutman, "A forgotten topological index," Journal of Mathematical Chemistry, vol. 53, no. 4, pp. 11841190, 2015.

[27] M. Javaid, M. U. Rehman, and J. Cao, "Topological indices of rhombus type silicate and oxide networks," Canadian Journal of Chemistry, vol. 95, no. 2, pp. 134-143, 2017.

[28] A. Vasilyev, "Upper and lower bounds of symmetric division deg index," Iranian Journal of Mathematical Chemistry, vol. 5, no. 2, pp. 91-98, 2014.

[29] M. Javaid, J. B. Liu, M. A. Rehman, and S. Wang, "On the certain topological indices of titania nanotube TiO2 [m, n]," Zeitschrift für Naturforschung A, vol. 72, no. 7, pp. 647-654, 2017.

[30] H. M. Awais, M. Jamal, and M. Javaid, “Topological properties of metal-organic frameworks," Main Group Metal Chemistry, vol. 43 , no. 1 , pp. $67-76,2020$.

[31] I. Gutman and N. Trinajstić, "Graph theory and molecular orbitals. total f-electron energy of alternant hydrocarbons," Chemical Physics Letters, vol. 17, no. 4, pp. 535-538, 1972.

[32] D. Amic, D. Belo, B. Lucic, S. Nikolic, and N. Trinajstic, "The vertex-connectivity index revisited," Journal of Chemical Information and Computer Sciences, vol. 38, no. 5, pp. 819822, 1998. 
[33] V. Lokesha and T. Deepika, "Symmetric division deg index of tricyclic and tetracyclic graphs," International journal of Science and Engineering Research, vol. 7, pp. 53-55, 2016.

[34] L. Zhong, "The harmonic index for graphs," Applied Mathematics Letters, vol. 25, no. 3, pp. 561-566, 2012.

[35] K. Pattabiraman, "Inverse sum indeg index of graphs," AKCE International Journal of Graphs and Combinatorics, vol. 15, no. 2, pp. 155-167, 2018.

[36] Y. Shi, M. Dehmer, X. Li, and I. Gutman, Graph Polynomials, CRC Press, Boca Raton, FL. USA, 2016.

[37] H. Yang, A. Q. Baig, W. Khalid, M. R. Farahani, and X. Zhang, "M-polynomial and topological indices of benzene ring embedded in P-type surface network," Journal of Chemistry, vol. 2019, Article ID 7297253, 9 pages, 2019.

[38] A. J. M. Khalaf, S. Hussain, D. Afzal, F. Afzal, and A. Maqbool, "M-polynomial and topological indices of book graph," Journal of Discrete Mathematical Sciences and Cryptography, vol. 23, no. 6, pp. 1217-1237, 2020.

[39] S. Mondal, M. Imran, N. De, and A. Pal, "Neighborhood M-polynomial of titanium compounds," Arabian Journal of Chemistry, vol. 14, no. 8, Article ID 103244, 2021. 